\title{
The Muon ATLAS MicroMegas Activity
}

\section{Georgios Tsipolitis*广}

National Technical University of Athens, Greece

E-mail: Yorgos.Tsipolitisecern.ch

\begin{abstract}
The luminosity upgrade of the Large Hadron Collider at CERN (sLHC) foresees a luminosity increase by a factor 5 compared to the LHC. To cope with the corresponding increase in background rates, the Muon System of the ATLAS experiment at CERN will likely need major changes in the very forward/backward region (the high rapidity region). The Muon ATLAS MicroMegas Activity (MAMMA) is focused on the development and testing of large-area muon detectors based on the resistive coating bulk-Micromegas technology as candidates for such an upgrade. This technology has undergone extensive tests with hadron beams at the CERN-SPS area, X-rays in the lab, as well as tests in a neutron beam at the TANDEM accelerator of the N.C.S.R. "Demokritos". In addition a set of prototype chambers have been installed in the ATLAS cavern and are taking data in real LHC conditions. Results on the performance of these chambers are presented.
\end{abstract}

The 2011 Europhysics Conference on High Energy Physics-HEP 2011,

July 21-27, 2011

Grenoble, Rhône-Alpes France

\footnotetext{
*Speaker.

${ }^{\dagger}$ Representing the MAMMA collaboration.
} 
The CERN Large Hadron Collider(LHC) started operation in 2009, providing proton-proton collissions at nominal luminosity of $10^{34} \mathrm{~cm}^{-2} \mathrm{~s}^{-1}$ at a center-of-mass energy of $14 \mathrm{TeV}$. The upgrade program for LHC is under study with the aim to increase the luminosity by a factor of 5 . With the luminosity upgrade of LHC, the rate of prompt muons and the background of photons and neutrons will increase proportionally. This particularly harsh background environment in the detectors imposes an upgrade of the present muon chambers in the End-cap inner and middle muon wheels of the ATLAS experiment. For the replacement, muon chambers based on the bulk-Micromegas [1] technology that combine precision measurement and triggering capability in the same detector are considered. The particularly harsh background environment in the detectors at the Large Hadron Collider at CERN for luminosities in excess of $10^{34} \mathrm{~cm}^{-2} \mathrm{~s}^{-1}$ places a number of severe constraints on the performance of such detectors. For example, count rates of up to $20 \mathrm{kHz} / \mathrm{cm}^{2}$ in the most unfavourable regions of the ATLAS muon system may have to be dealt with [2]. Less than $10 \%$ of this rate is expected to come from muons, approximately $20 \%$ from protons and pions, the rest, in the approximate proportion of 2:1, stems from photon and neutron interactions. In particular the latter are of concern. Neutrons interacting in the chambers create slowly moving recoils from elastic scattering and/or low-energy hadronic debris from nuclear breakup. They both are heavily ionizing and lead to large energy deposits in the muon chambers with the risk of sparking. In addition to neutrons, also charged-hadron interactions can create low-energy hadronic debris, as discussed for example in Ref. [3]. The specific properties of micromegas chambers, with a very thin amplification region, make them particularly vulnerable to sparking. Sparks occur when the total number of electrons in the avalanche reaches values of a few $10^{7}$ (Raether limit [4]). High detection efficiency for minimum ionizing muons calls for gas amplification factors of the order of $10^{4}$. Therefore, ionization processes producing more than 1000 electrons over distances comparable to the typical lateral extent of an avalanche (a few $100 \mu \mathrm{m}$ ) carry the risk of sparking, see for example Ref. [5]. Such ionization levels are easily reached by low-energy alpha-particles or slowly moving charged debris from neutron (or other) inter- actions in the detector gas or detector materials. Sparks may damage the detector and read out electronics and/or lead to large dead times as a resultof HV breakdown. Initial test with normal bulk-micromegas chambers, both in charged hadron and neutron beams, showed this limitation. This problem is solved with a new type of resistive micromegas detector. It is a bulk-micromegas structure built on top of a printed circuit board (PCB) with $18 \mathrm{~mm}$ thick $\mathrm{Cu}$ readout strips covered by a resistive protection layer. More details on the detector construction may be found in Ref. [6]. The chambers have undergone extensive tests in the lab with a ${ }^{55} \mathrm{Fe}$ source and a $8 \mathrm{keV} \mathrm{Cu} \mathrm{X}$-ray gun. In addition several tests with pion and neutron beams were performed with the chamber running very smoothely. This is demonstrated in Figure 1. It shows the monitored $\mathrm{HV}$ and the currents for a normal bulk micromegas and a resistive micromegas detector. Both chambers were operated with a $\mathrm{A} r: \mathrm{CO}_{2}$ 93:7 gas mixture and were exposed in the same neutron flux for different mesh HV settings. The energy of the neutrons was 5.5 MeV. In the non-resistive chamber the mesh HV broke down as soon as the neutron beam was switched on. The currents to recharge the mesh exceeded the current limitation of the power supply which was set to $2 \mathrm{~mA}$; HV drops of the order of $50 \mathrm{~V}$ were observed. For resistive micromegas detector no HV breakdown is observed. The currents do not exceed $200 \mathrm{nA}$ for a mesh HV of $590 \mathrm{~V}$, corresponding to an effective gas gain of 12,000. The few high current points in Figure 1 correspond to the currents during HV ramp up. Similar behaviour was observed also in a pion 


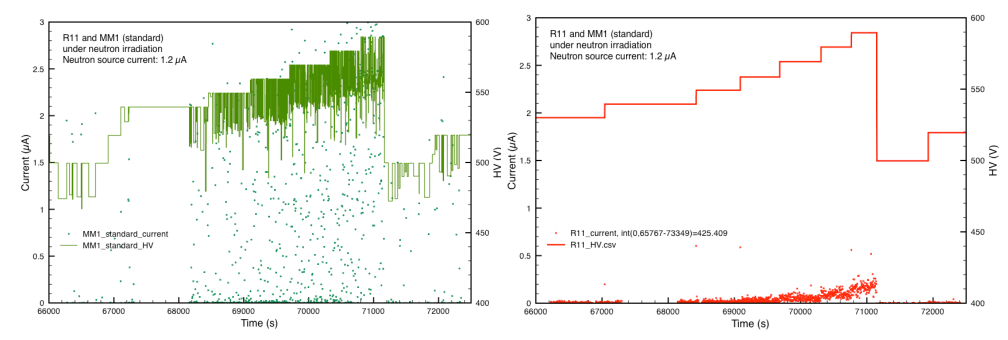

Figure 1: The HV scan and recorded current for a normal bulk-micromegas (left) and a resistive micromegas chamber in a neutron flux of $1.5 \times 10^{6} \mathrm{ncm}^{-2} \mathrm{~s}^{-1}$.

beam of $120 \mathrm{GeV} / \mathrm{c}$. Figure 2 number interactions and the number of sparks per incident neutron as a function of the gain of the chamber. Two different gas mixtures of $\mathrm{A} r: \mathrm{CO}_{2}$ were used (80:20 and 93:7) and the neutron flux was $1.5 \times 10^{6} \mathrm{ncm}^{-2} \mathrm{~s}^{-1}$.

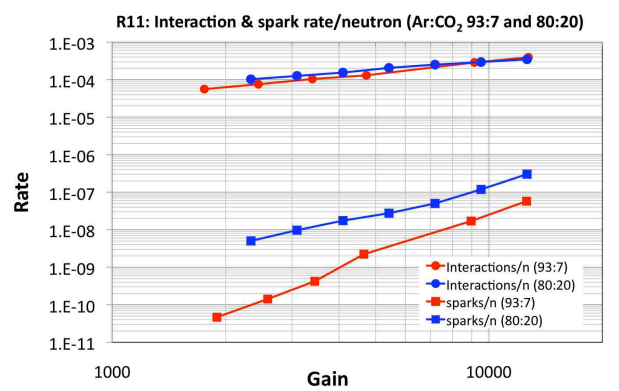

Figure 2: The number interactions (circles) and the number of sparks per incident neutron (squares) as a function of the gain of the chamber for two different gas mixtures of $\mathrm{Ar}: \mathrm{CO}_{2}, 80: 20$ (blue) and 93:7 (red) and a neutron flux of $1.5 \times 10^{6} \mathrm{ncm}^{-2} \mathrm{~s}^{-1}$.

In conclusion, a spark-resistant bulk-micromegas chambers is constructed by adding above the readout strips a layer of resistive strips. The resistive strips are separated by an insulating layer from the readout strips and individually grounded through a large resistance. The chambers perform well with photons from a ${ }^{55} \mathrm{Fe}$ source and a $8 \mathrm{keV} \mathrm{Cu} \mathrm{X-ray} \mathrm{gun,} \mathrm{as} \mathrm{well} \mathrm{as} \mathrm{with} 120 \mathrm{GeV}$ pions. The chambers reach gas gains up to 30000 and can be operated comfortably at gains of $10^{4}$. The chambers were also operated stably under neutron fluxes of $1.5 \times 10^{6} \mathrm{ncm}^{-2} \mathrm{~s}^{-1}$. Sparks are no longer limiting the performance of micromegas chamber.

\section{References}

[1] I. Giomataris, et al., Nucl. Instr. and Meth. A 376 (1996) 29., I. Giomataris, et al., Nucl. Instr. and Meth. A 560 (2006) 405.

[2] G. Aad, et al., J. Inst. 3 (2008) S08003.

[3] S. Procureur, et al., Nucl. Instr. and Meth. A 621 (2010) 177.

[4] H. Raether, Z. Phys. 112 (1939) 464.

[5] V. Peskov, et al., J. Inst. 5 (2010) P11004.

[6] T. Alexopoulos et. al., Nucl. Instr. and Meth. A 640 (2011) 110. 